Article

\title{
Towards a Valorization of Corn Bioethanol Side Streams: Chemical Characterization of Post Fermentation Corn Oil and Thin Stillage
}

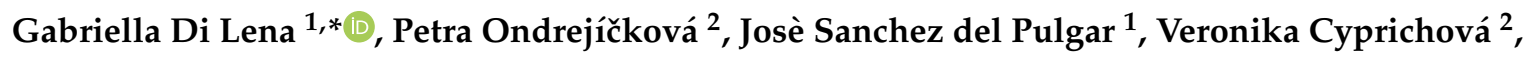 \\ Tomáš Ježovič ${ }^{2}$, Massimo Lucarini ${ }^{1}$, Ginevra Lombardi Boccia ${ }^{1}$, Stefano Ferrari Nicoli ${ }^{1}$, \\ Paolo Gabrielli ${ }^{1}$, Altero Aguzzi ${ }^{1}$, Irene Casini ${ }^{1}$ and Roberto Caproni ${ }^{1}$ \\ 1 CREA Research Centre for Food and Nutrition, Via Ardeatina 546, 00178 Rome, Italy; \\ jose.sanchezdelpulgar@crea.gov.it (J.S.d.P.); massimo.lucarini@crea.gov.it (M.L.); \\ g.lombardiboccia@crea.gov.it (G.L.B.); stefano.nicoli@crea.gov.it (S.F.N.); paolo.gabrielli@crea.gov.it (P.G.); \\ altero.aguzzi@crea.gov.it (A.A.); irene.casini@crea.gov.it (I.C.); roberto.caproni@crea.gov.it (R.C.) \\ 2 ENVIRAL a.s., Trnavská Cesta, 92041 Leopoldov, Slovakia; Ondrejickova@enviengroup.eu (P.O.); \\ Cyprichova@enviengroup.eu (V.C.); Jezovic@enviral.sk (T.J.) \\ * Correspondence: gabriella.dilena@crea.gov.it; Tel.: +39-06-51494445
}

Academic Editors: Maria Beatriz Prior Pinto Oliveira and Rita Carneiro Alves Received: 29 June 2020; Accepted: 30 July 2020; Published: 3 August 2020

\begin{abstract}
First-generation biofuel biorefineries may be a starting point for the development of new value chains, as their by-products and side streams retain nutrients and valuable molecules that may be recovered and valorized for high-value applications. This study provides a chemical characterization of post-fermentation corn oil and thin stillage, side streams of dry-grind corn bioethanol production, in view of their valorization. An overall long-term study was conducted on the two co-products collected over 1 year from a bioethanol plant. Water content, acid value, sedimentation, mineral composition, and fatty acid profiles were analyzed on post-fermentation corn oil. Results highlighted that its acid value was high (19.72-24.29 $\mathrm{mg} \mathrm{KOH} / \mathrm{g})$, indicating high levels of free fatty acids, but stable over the year due to standardized operating conditions. The fatty acid profile was that typical of corn oil, with a prevalence of linoleic (54-59\% of total fatty acids) over oleic (23-27\%) and palmitic (12-17\%) acids. Macronutrients, fatty acid, and mineral profiles were investigated in thin stillage. Results revealed the acidic $\mathrm{pH}(4.05-4.68)$ and high dilution (90-93\% water) of this side stream. The dry mass was composed of fats (19-30\%), proteins (8.8-12.8\%), ash (8.7-9.5\%), and fiber (7.3-9.8\%). The concomitant presence of a variegate complex of molecules of nutritional interest in corn bioethanol co-products, with several potential high-value market applications, make the perspective of their recovery a promising strategy to create new cross-sector interconnections according to circular economy principles.
\end{abstract}

Keywords: corn bioethanol side streams; bioethanol co-products; post-fermentation corn oil; thin stillage; by-products valorization; chemical composition; macronutrients; minerals; fatty acids

\section{Introduction}

With the increasing world population, industrial expansion, and growth of global energy demand, the consumption of fossil fuels has become unsustainable, leading to depletion of resources and global warming.

The promotion of renewable energy is an essential part of the European energy policy, as recognized in the Treaty on the Functioning of the European Union [1] and in the implementation of the Energy Union Strategic Framework [2,3]. The legislative framework for production and usage of biofuels 
is provided by regulations promoting the use of energy from renewable sources and establishing sustainability criteria in order to reduce greenhouse gas (GHG) emissions and limit global land conversion for biofuel feedstock production [4-6].

Bioethanol is the second largest contributor of renewable energy sources to the transport sector and represents $20 \%$ of biofuels in Europe [7]. It is mainly produced from crop-based feedstock, with corn representing $43 \%$ of feedstocks, followed by wheat (26\%), sugar-beet $(21 \%)$, and other cereals and starch-rich crops (6\%) [8]. In Europe, the installed grain-to-ethanol production capacity has steadily increased in the last few years, reaching 8901 million L in 2018 with an average of $70 \%$ GHG savings compared to fossil fuels [9]. Further increments are expected in the future; yet, to increase the competitiveness of biofuels, a significant reduction of their production costs is necessary. A promising approach is the valorization of co-products and side streams with the creation of biofuel-driven integrated biorefineries, opening opportunities for new bio-based value chains. This approach is in line with the circular economy principles aiming at ensuring high quality, functional, and safe products to all, while reducing carbon and environmental footprints $[10,11]$.

At the same time, with the perspective of a growing world population reaching 9 billion by 2050, sustainable food security, defined as the availability of food providing a healthy and nutritious diet to all, is an emerging issue. Agri-food by-products and agro-industrial wastes, being still rich in nutritious and functional ingredients, should be exploited as sustainable sources of nutrients. Within this context, first-generation biofuel biorefineries may be a starting key point for new sustainable biobased value chains, as their by-products and side streams still retain series of nutrients and valuable molecules that, if properly recovered and valorized, may open new perspectives for integrated biorefinery systems with the concomitant advantage of increasing the competitiveness and maximizing the efficiency of the bio-fuel production process.

The dry grind corn bioethanol process entails several key steps including enzymatic liquefaction and saccharification, releasing glucose from starch, followed by fermentation by means of the yeast Saccharomyces cerevisiae and further distillation. Two investigated co-products of dry grind corn bioethanol production are post-fermentation corn oil and thin stillage. Post fermentation corn oil differs from pressed corn oil by means of corn feedstock being processed by enzymatic hydrolysis followed by a fermentation process and subsequent distillation when being isolated from residual whole stillage by decanter. Post-fermentation corn oil, separated from corn syrup by centrifugation, may be utilized for industrial applications such as bio-diesel production if there is an adjacent biodiesel plant. Prior to this application, the recovery process and valorization of present bioactive compounds (sterols, vitamins, phenolics) should be developed and applied for their high-value applications (food, nutraceuticals, cosmetics). The thin stillage stream is a supernatant liquid fraction generated in large amounts after centrifugation of thick/whole stillage. It consists primarily of suspended and dissolved particles and nutrients originating from spent grains as well as yeast cells; therefore, it is still rich in compounds that deserve to be recovered. Currently, the significant fraction of thin stillage is used to produce animal feed distiller's dried grains with solubles (DDGS) by multiple energy-intensive evaporations. However, the high cost of concentrating thin stillage and its relatively low nutritional value suggest other applications. One currently applicable use of thin stillage is as a backset water for the liquefaction step preceding fermentation in bioethanol plants $[12,13]$. Nonetheless, both solutions are low-purpose usages, not really valorizing the full set of components present in it. Alternative strategies to valorize thin stillage have been studied, like the use as a growth medium for filamentous fungi $[14,15]$ or algae $[16,17]$ finalized to obtain high-value biomass and bio-products. Notwithstanding, the extraction and fractionation of valuable compounds present in thin stillage by means of suitable technologies and their utilization as ingredients for high-value markets could represent the most ambitious and profitable way to valorize this side stream.

The EXCornsEED Project, financed within the European Union's Horizon 2020-BBI-JU Program, has the main goal of developing and validating an integrated process of innovative and highly sustainable technologies to recover proteins and other valuable compounds from side streams of 
first-generation biofuel biorefineries and to apply them as ingredients for high-value market products. The first step of the Project has been to assess the chemical composition of side streams in order to evaluate their valorization potentials. Post-fermentation corn oil and thin stillage provided by a dry-grind corn bioethanol plant have been the main objects of this study (Figure S1).

Literature studies by other authors have highlighted the organic and inorganic compounds present in grain-based ethanol thin stillage and post fermentation corn oil [12,18-23] but, to our best knowledge, no manuscript reports on a systematic study for an extensive period of time focused on chemical characteristics of co-products of a commercial dry-grind corn-based bioethanol facility.

We herewith report the results of a study, conducted in the framework of the EXCornsEED Project, focused on the assessment of components of nutritional interest (macronutrients, fatty acids, and minerals) in post-fermentation corn oil and thin stillage collected over a one-year period. The aim of this study was to determine the composition of selected side-streams and to provide comprehensive information about their chemical profile to explore their full valorization potentialities.

\section{Results and Discussion}

\subsection{Corn Oil}

Compositional parameters of the post-fermentation corn oil side stream from bioethanol production play an important part in the subsequent non-edible application for biodiesel production, therefore, corn oil is being routinely tested on a daily basis for output parameters at ENVIRAL premises.

The water residue of vegetable oils is considered a factor diminishing the oil quality and stability because of the physical, chemical, and microbial changes that it may trigger. At the same time, in view of biodiesel production, water is considered an oil contaminant, reducing the yield of the transesterification process [24]. Therefore, the water content together with total impurities are monitored in the oil before its further processing. As shown in Table 1, the levels of water and total contaminants on a monthly basis collected over a one-year period, were below the internal limit of $2 \% w / w$.

Some parameters, like the sedimentation value, might vary according to seasonal changes, being inversely related to the outer temperature associated to the sedimentation tank surroundings. During the second half of the evaluation year, sedimentation of corn oil was also monitored. From the results shown in Table 1, an increasing trend of the values starting from the summer to winter months was observed. This can be connected with seasonal changes of the outer temperature, which affects the temperature of tanks, where the level of sedimentation in the tanks increases with the lower temperatures.

In line with the high temperatures reached during the biotech process, the acid value of post-fermentation corn oil was quite remarkable (19.72-24.29 $\mathrm{mg} \mathrm{KOH} / \mathrm{g})$, indicating high levels of free fatty acids. This is a critical parameter, negatively affecting the oxidative stability of the oil and also the yield of the transesterification reaction. In fact, free fatty acids in the oil are converted to soaps during base-catalyzed esterification, thus lowering the yield of biodiesel [25]. The average acid values of corn oil, oscillating in the narrow range $20-25 \mathrm{mg} \mathrm{KOH} / \mathrm{g}$, are an indication of stable operating conditions. These values are expected in an industrial oil that underwent severe heat treatments and are comparable to those reported by other authors [22].

Crude vegetable oils contain phosphorus in the form of phospholipids, which are also referred to as gums. As the higher levels of phosphorus in oils have a negative effect on biodiesel yield, this impurity is removed during feedstock pre-treatment in the degumming process. The level of phosphorus is also strictly controlled in the final biodiesel as it increases the exhaust emissions [26]. The results of the average phosphorus content in corn oil confirmed that the values comply with ENVIRAL's internal limit of $30 \mathrm{mg} / \mathrm{kg}$ that corn oil has to meet for its subsequent utilization as a feedstock in an adjacent biodiesel plant. 
Table 1. Chemical characteristics of corn oil from a dry-grind corn ethanol plant. Data obtained by ENVIRAL on samples collected over a one-year period *.

\begin{tabular}{|c|c|c|c|c|c|c|c|c|c|c|c|c|c|}
\hline & Lot 1 & Lot 2 & Lot 3 & Lot 4 & Lot 5 & Lot 6 & Lot 7 & Lot 8 & Lot 9 & mean & sd & $\min$ & $\max$ \\
\hline Water content $(\mathrm{g} / 100 \mathrm{~g})$ & $0.51 \pm 0.07$ & $0.41 \pm 0.07$ & $0.45 \pm 0.08$ & $0.44 \pm 0.07$ & $0.44 \pm 0.08$ & $0.34 \pm 0.02$ & $0.36 \pm 0.03$ & $0.46 \pm 0.23$ & $0.43 \pm 0.09$ & 0.43 & 0.05 & 0.34 & 0.51 \\
\hline Sedimentation (vol \%) & $5.88 \pm 2.09$ & $7.29 \pm 3.96$ & $13.88 \pm 4.64$ & $8.8 \pm 0.82$ & $7.14 \pm 1.89$ & $6.50 \pm 2.51$ & $9.20 \pm 2.09$ & $11.8 \pm 3.24$ & $13.0 \pm 2.53$ & 9.28 & 2.95 & 5.88 & 13.88 \\
\hline $\begin{array}{l}\text { Total contamination } \\
(\mathrm{g} / 100 \mathrm{~g})\end{array}$ & $0.01 \pm 0.01$ & $0.06 \pm 0.01$ & $0.01 \pm 0.01$ & $0.01 \pm 0.01$ & $0.01 \pm 0.00$ & $0.01 \pm 0.00$ & $0.28 \pm 0.03$ & $0.19 \pm 0.04$ & $0.01 \pm 0.01$ & 0.06 & 0.10 & 0.01 & 0.28 \\
\hline $\begin{array}{l}\text { Acid value }(\mathrm{mg} \mathrm{KOH} / \mathrm{g}) \\
\text { Minerals: }\end{array}$ & $21.02 \pm 1.26$ & $24.29 \pm 1.51$ & $21.33 \pm 1.91$ & $19.72 \pm 0.78$ & $21.79 \pm 1.72$ & $20.90 \pm 0.79$ & $21.9 \pm 1.95$ & $22.86 \pm 0.77$ & $20.75 \pm 1.21$ & 21.62 & 1.33 & 19.72 & 24.29 \\
\hline $\mathrm{P}(\mathrm{mg} / \mathrm{kg})$ & $7.5 \pm 1.6$ & $6.18 \pm 1.08$ & $18.81 \pm 11.34$ & $9.78 \pm 5.77$ & $34.45 \pm 9.44$ & $10.6 \pm 2.2$ & $9.75 \pm 3.65$ & $3.52 \pm 1.28$ & $10.87 \pm 2.72$ & 12.38 & 4.34 & 3.52 & 34.45 \\
\hline $\mathrm{K}(\mathrm{mg} / \mathrm{kg})$ & $3.27 \pm 1.06$ & $1.61 \pm 0.40$ & $11.83 \pm 7.42$ & $7.10 \pm 2.94$ & $6.33 \pm 3.64$ & $<1$ & $7.40 \pm 3.58$ & $1.66 \pm 0.41$ & $15.53 \pm 6.97$ & - & - & $<1$ & 15.53 \\
\hline $\mathrm{Na}(\mathrm{mg} /$ & $0.57 \pm 0.31$ & $1.99 \pm 1.20$ & $<1$ & $<1$ & $2.57 \pm 1.66$ & $2.78 \pm 1.66$ & $2.94 \pm 1.41$ & $1.02 \pm 0.42$ & $4.74 \pm 2.53$ & - & - & 0.57 & 4.74 \\
\hline $\operatorname{Mg}(\mathrm{n}$ & $0.84 \pm 0.34$ & $<1$ & $<1$ & $<1$ & $1.63 \pm 0.47$ & $<1$ & $1.36 \pm 0.87$ & $<1$ & $1.37 \pm 1.17$ & - & - & 0.84 & 1.63 \\
\hline $\mathrm{Ca}(\mathrm{mg} / \mathrm{kg})$ & $0.05 \pm 0.03$ & $<1$ & $<1$ & $<1$ & $<1$ & $<1$ & $<1$ & $<1$ & $<1$ & - & - & 0.05 & $<1$ \\
\hline
\end{tabular}

* data for each monthly lot represent mean \pm sd of triplicate measurements. Details on the origin of corn feedstock and on lot timings are provided in Materials and Methods and in Table S1. 
Calcium, magnesium, sodium, and potassium are inorganic contaminants that, when present in biodiesel, may negatively affect engine performance and contribute to biodiesel decomposition [27]. As shown in Table 1, the contents of calcium and magnesium were almost constant during the year with fluctuating values of potassium observed but still meeting internal limits and having no impact on final biodiesel product properties.

The fatty acid profile of individual lots of post-fermentation corn oil is reported in Table 2. The profile observed was that typical of corn oil [28,29], with linoleic acid (C18:2 n-6) as the prevalent fatty acid (54-59\% of total fatty acids) followed by oleic (C18:1 n-9, 23-27\%) and palmitic (C16:0, $12-17 \%)$ acids. Minor amounts of stearic acid (C18:0, 1-2\%) and $\alpha$-linolenic acid (C18:2 n-6, 1.6-2.7\%) and trace amounts of other saturated and unsaturated fatty acids were also detected.

Along with methyl esters, the mass spectrum analysis enabled the identification of minor amounts $(5-10 \%)$ of ethyl esters of fatty acids like palmitic and linoleic acids, presumably already present in the original sample before transesterification as a result of yeast metabolism. The values reported in Table 2 for palmitic and linoleic acids are the sum of ethyl and methyl esters. The comparison of the different lots of corn oil examined showed a low variability of the fatty acid profile, as indicated from the coefficient of variation (CV) of major compounds $(2.5 \%, 6.1 \%$, and $13.9 \%$ for linoleic, oleic, and palmitic acids, respectively). This indicates a comparable quality of corn feedstock and standardized industrial processing conditions. The profile observed is in accordance with that reported by other authors although, to the best of our knowledge, no one has reported on the presence of ethyl esters of fatty acids until now [20].

\subsection{Thin Stillage}

The proximate composition of different lots of thin stillage collected over the period July 2018-June 2019 , expressed on a wet mass and on a dry mass basis, is reported in Table 3 . Thin stillage, currently utilized after condensation together with DDGS as a feed ingredient, is characterized by an acidic $\mathrm{pH}$ (4.05-4.68) and a very high dilution (about 90-93\% water content). $\mathrm{pH}$ is within the range of internal limits of $\mathrm{pH}$ 3.7-4.7. The acidic $\mathrm{pH}$ is mainly attributable to the presence of organic acids, mainly acetic, lactic, and propionic acids resulting from the fermentation process $[18,23]$. The dry matter value in the series of samples analyzed was almost stable (6.8-9.9\%), indicating constant conditions of the biotech process. Nitrogen analyses evidenced high percentages of nonprotein nitrogen (NPN), corresponding to about $50 \%$ of total $\mathrm{N}$ in all thin stillage batches. NPN compounds in thin stillage arise from corn residues, yeast metabolites, and from the urea added as a $\mathrm{N}$ source for yeasts during the bioethanol production process. In consideration of the high NPN content of thin stillage, in place of the common crude protein content calculated on the base of the total $\mathrm{N}$ content, that would highly overestimate proteins, we calculated the true protein content, which better reflects the particular composition of thin stillage.

The dry matter was composed of fats (19-30\% dry mass), true proteins (8.8-12.8\% dry mass), calculated by subtracting nonprotein $\mathrm{N}$ to total $\mathrm{N}$, ash (7.8-11.4\% dry mass), and total dietary fiber (8.4-24.4\% dry mass). The remaining fraction (36-53\% dry mass) may be ascribed to oligosaccharides, unfermented sugars, organic acids, and glycerol left after fermentation, as indicated in the literature [23]. Minor variations observed in the thin stillage composition can be caused by seasonal differences in maize composition and also by slight changes in process parameters such as acid addition rates, enzyme loadings, temperatures, and fermentation conditions.

The mineral and trace element profiles of the different lots of thin stillage analyzed are reported in Table 4. Thin stillage showed a prevalence of potassium (1.8-2.8\% dry mass) and phosphorus (1.3-1.8\% dry mass) over sodium (0.4-0.9\% dry mass) and magnesium (0.5-0.8\% dry mass) and minor amounts of calcium (0.06-0.09\% dry mass). Iron, zinc, manganese, and copper were detected at very low levels. 
Table 2. Fatty acid profile of post-fermentation corn oil from the dry-grind corn ethanol plant. Mean, standard deviation, and range of values observed over a one-year period $(n=8) *$. Values are expressed as percent of total fatty acids.

\begin{tabular}{|c|c|c|c|c|c|c|c|c|c|c|c|c|}
\hline & Lot 1 & Lot 2 & Lot 3 & Lot 4 & Lot 5 & Lot 6 & Lot 7 & Lot 8 & mean & $\mathrm{sd}$ & $\min$ & $\max$ \\
\hline & \multicolumn{12}{|c|}{$\%$ of total fatty acids } \\
\hline Lauric acid (C12:0) & $0.00 \pm 0.00$ & $0.02 \pm 0.01$ & $0.02 \pm 0.01$ & $0.00 \pm 0.00$ & $0.00 \pm 0.00$ & $0.00 \pm 0.00$ & $0.00 \pm 0.00$ & $0.00 \pm 0.00$ & 0.01 & 0.01 & 0.00 & 0.02 \\
\hline Myristic acid (C14:0) & $0.00 \pm 0.00$ & $0.04 \pm 0.02$ & $0.05 \pm 0.02$ & $0.03 \pm 0.01$ & $0.08 \pm 0.03$ & $0.12 \pm 0.03$ & $0.12 \pm 0.02$ & $0.00 \pm 0.00$ & 0.07 & 0.04 & 0.03 & 0.12 \\
\hline Pentadecylic acid (C15:0) & $0.00 \pm 0.00$ & $0.00 \pm 0.00$ & $0.00 \pm 0.00$ & $0.00 \pm .0 .00$ & $0.00 \pm 0.00$ & $0.00 \pm 0.00$ & $0.00 \pm 0.00$ & $0.09 \pm 0.04$ & 0.01 & 0.03 & 0.00 & 0.09 \\
\hline Palmitic acid (C16:0) & $12.11 \pm 0.30$ & $11.56 \pm 0.34$ & $15.11 \pm 1.10$ & $14.27 \pm 2.13$ & $15.38 \pm 0.57$ & $16.31 \pm 0.48$ & $17.33 \pm 1.85$ & $13.39 \pm 1.03$ & 14.43 & 2.00 & 11.56 & 17.33 \\
\hline Palmitoleic acid (C16:1 n-7) & $0.00 \pm 0.00$ & $0.08 \pm 0.00$ & $0.05 \pm 0.02$ & $0.05 \pm 0.01$ & $0.00 \pm 0.00$ & $0.00 \pm 0.00$ & $0.00 \pm 0.00$ & $0.00 \pm 0.00$ & 0.06 & 0.02 & 0.05 & 0.08 \\
\hline Margaric acid (C17:0) & $0.04 \pm 0.01$ & $0.05 \pm 0.01$ & $0.05 \pm 0.02$ & $0.00 \pm 0.00$ & $0.00 \pm 0.00$ & $0.00 \pm 0.00$ & $0.00 \pm 0.00$ & $0.00 \pm 0.00$ & 0.05 & 0.01 & 0.04 & 0.05 \\
\hline Stearic acid (C18:0) & $1.40 \pm 0.08$ & $1.47 \pm 0.09$ & $1.91 \pm 0.34$ & $1.84 \pm 0.66$ & $1.61 \pm 0.05$ & $1.17 \pm 0.10$ & $1.06 \pm 0.71$ & $1.41 \pm 0.03$ & 1.48 & 0.29 & 1.06 & 1.91 \\
\hline Oleic acid (C18:1 n-9) & $26.44 \pm 1.78$ & $25.99 \pm 1.80$ & $23.44 \pm 0.43$ & $23.02 \pm 0.78$ & $23.13 \pm 0.72$ & $25.20 \pm 1.32$ & $25.37 \pm 2.15$ & $26.85 \pm 1.07$ & 24.93 & 1.53 & 23.02 & 26.85 \\
\hline Linoleic acid (C18:2n-6) & $57.99 \pm 1.40$ & $58.63 \pm 1.41$ & $57.30 \pm 0.75$ & $57.06 \pm 2.51$ & $57.70 \pm 0.29$ & $55.50 \pm 0.81$ & $54.12 \pm 0.48$ & $56.96 \pm 0.27$ & 56.91 & 1.45 & 54.12 & 58.63 \\
\hline$\alpha$-Linolenic acid (C18:3 n-3) & $1.78 \pm 0.30$ & $1.78 \pm 0.31$ & $1.78 \pm 0.38$ & $2.68 \pm 1.63$ & $1.98 \pm 0.10$ & $1.64 \pm 0.09$ & $1.70 \pm 0.05$ & $1.73 \pm 0.07$ & 1.88 & 0.34 & 1.64 & 2.68 \\
\hline Arachidic acid (C20:0) & $0.18 \pm 0.05$ & $0.16 \pm 0.07$ & $0.07 \pm 0.04$ & $0.00 \pm 0.00$ & $0.11 \pm 0.02$ & $0.00 \pm 0.00$ & $0.34 \pm 0.08$ & $0.27 \pm 0.10$ & 0.19 & 0.10 & 0.07 & 0.34 \\
\hline Gondoic acid (C20:1 n-9) & $0.29 \pm 0.03$ & $0.21 \pm 0.06$ & $0.27 \pm 0.02$ & $0.00 \pm 0.00$ & $0.18 \pm 0.03$ & $0.00 \pm 0.00$ & $0.00 \pm 0.00$ & $0.00 \pm 0.00$ & 0.24 & 0.05 & 0.18 & 0.29 \\
\hline Eicosadienoic acid (C20:2n-6) & - & - & - & - & - & 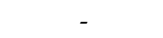 & - & - & - & - & - & - \\
\hline Behenic acid (C22:0) & - & - & - & - & 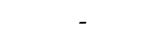 & 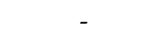 & - & - & - & - & - & - \\
\hline Total SFA & $13.73 \pm 0.30$ & $13.27 \pm 0.36$ & $17.18 \pm 1.10$ & $16.14 \pm 1.64$ & $17.13 \pm 0.61$ & $17.67 \pm 0.49$ & $18.81 \pm 2.51$ & $15.14 \pm 1.06$ & 16.13 & 1.95 & 13.27 & 18.81 \\
\hline Total MUFA & $26.73 \pm 1.70$ & $26.26 \pm 1.80$ & $23.67 \pm 0.53$ & $23.04 \pm 0.75$ & $23.19 \pm 0.69$ & $25.20 \pm 1.32$ & $25.37 \pm 2.15$ & $26.85 \pm 1.07$ & 25.04 & 1.56 & 23.04 & 26.85 \\
\hline Total PUFA & $59.77 \pm 1.60$ & $60.40 \pm 1.66$ & $59.08 \pm 0.64$ & $59.74 \pm 0.88$ & $59.67 \pm 0.23$ & $57.14 \pm 0.90$ & $55.82 \pm 0.53$ & $58.12 \pm 1.08$ & 58.72 & 1.57 & 55.82 & 60.40 \\
\hline Total n-6 PUFA & $57.99 \pm 0.50$ & $58.63 \pm 1.41$ & $57.30 \pm 0.75$ & $57.06 \pm 2.51$ & $57.70 \pm 0.29$ & $55.50 \pm 0.81$ & $54.14 \pm 0.48$ & $56.96 \pm 0.27$ & 56.91 & 1.45 & 54.12 & 58.63 \\
\hline Total n-3 PUFA & $1.78 \pm 0.40$ & $1.78 \pm 0.31$ & $1.78 \pm 0.38$ & $2.68 \pm 1.63$ & $1.98 \pm 0.10$ & $1.64 \pm 0.09$ & $1.70 \pm 0.05$ & $1.73 \pm 0.07$ & 1.88 & 0.34 & 1.64 & 2.68 \\
\hline$n-6 / n-3$ PUFA ratio & $32.58 \pm 1.50$ & $33.50 \pm 4.79$ & $33.17 \pm 6.89$ & $26.54 \pm 13.00$ & $29.26 \pm 1.58$ & $33.85 \pm 1.39$ & $31.81 \pm 0.72$ & $32.96 \pm 1.02$ & 31.71 & 2.53 & 26.54 & 33.85 \\
\hline
\end{tabular}

* data for each monthly 
Table 3. Chemical composition of thin stillage derived from the dry-grind corn ethanol plant. Mean, standard deviation, and range of values observed over a one-year period $(n=9)$ *.

\begin{tabular}{|c|c|c|c|c|c|c|c|c|c|c|c|c|c|}
\hline & Lot 1 & Lot 2 & Lot 3 & Lot 4 & Lot 5 & Lot 6 & Lot 7 & Lot 8 & Lot 9 & mean & sd & $\min$ & $\max$ \\
\hline $\mathrm{pH}$ & \multicolumn{13}{|c|}{ g $100 \mathrm{~g}^{-1}$ wet mass } \\
\hline Dry matter & $7.69 \pm 0.05$ & $7.99 \pm 0.05$ & $7.86 \pm 0.01$ & $8.44 \pm 0.01$ & $6.81 \pm 0.07$ & $7.96 \pm 0.02$ & $8.74 \pm 0.07$ & $9.93 \pm 0.08$ & $8.57 \pm 0.03$ & 8.22 & 0.86 & 6.81 & 9.93 \\
\hline Water content & $92.32 \pm 0.02$ & $92.01 \pm 0.05$ & $92.14 \pm 0.01$ & $91.56 \pm 0.01$ & $93.19 \pm 0.07$ & $92.04 \pm 0.02$ & $91.26 \pm 0.07$ & $90.07 \pm 0.08$ & $91.43 \pm 0.03$ & 91.78 & 0.86 & 90.07 & 93.19 \\
\hline Total N & $0.22 \pm 0.01$ & $0.23 \pm 0.03$ & $0.24 \pm 0.01$ & $0.32 \pm 0.02$ & $0.27 \pm 0.01$ & $0.27 \pm 0.01$ & $0.27 \pm 0.01$ & $0.32 \pm 0.00$ & $0.26 \pm 0.01$ & 0.27 & 0.03 & 0.22 & 0.32 \\
\hline Nonprotein N & $0.11 \pm 0.01$ & $0.12 \pm 0.01$ & $0.12 \pm 0.01$ & $0.15 \pm 0.01$ & $0.13 \pm 0.00$ & $0.13 \pm 0.01$ & $0.10 \pm 0.00$ & $0.13 \pm 0.00$ & $0.12 \pm 0.00$ & 0.12 & 0.01 & 0.10 & 0.15 \\
\hline True Protein & $0.68 \pm 0.09$ & $0.71 \pm 0.16$ & $0.78 \pm 0.01$ & $1.07 \pm 0.13$ & $0.87 \pm 0.08$ & $0.83 \pm 0.08$ & $1.03 \pm 0.02$ & $1.19 \pm 0.02$ & $0.91 \pm 0.03$ & 0.90 & 0.17 & 0.68 & 1.19 \\
\hline Ash & $0.73 \pm 0.05$ & $0.69 \pm 0.02$ & $0.71 \pm 0.01$ & $0.75 \pm 0.02$ & $0.78 \pm 0.01$ & $0.81 \pm 0.01$ & $0.73 \pm 0.03$ & $0.77 \pm 0.02$ & $0.69 \pm 0.02$ & 0.74 & 0.04 & 0.69 & 0.81 \\
\hline Crude fat & $2.33 \pm 0.02$ & $1.52 \pm 0.06$ & $1.83 \pm 0.02$ & $1.81 \pm 0.01$ & $1.46 \pm 0.05$ & $1.64 \pm 0.02$ & $1.67 \pm 0.12$ & - & - & 1.75 & 0.29 & 1.46 & 2.33 \\
\hline Total dietary fiber & $0.65 \pm 0.08$ & $0.83 \pm 0.10$ & $0.95 \pm 0.06$ & $1.06 \pm 0.01$ & $1.21 \pm 0.45$ & $1.31 \pm 0.54$ & $2.14 \pm 0.08$ & $2.03 \pm 0.06$ & - & 1.27 & 0.54 & 0.65 & 2.14 \\
\hline Other solubles ** & $3.29 \pm 0.13$ & $4.24 \pm 0.29$ & $3.57 \pm 0.11$ & $3.82 \pm 0.03$ & $2.51 \pm 0.50$ & $3.43 \pm 0.60$ & $3.14 \pm 0.24$ & - & - & 3.43 & 0.54 & 2.51 & 4.24 \\
\hline & \multicolumn{13}{|c|}{ g $100 \mathrm{~g}^{-1}$ dry mass } \\
\hline $\begin{array}{l}\text { Dry matter } \\
\text { Total N }\end{array}$ & $\begin{array}{c}100 \\
2.87 \pm 0.11\end{array}$ & $\begin{array}{c}100 \\
2.88 \pm 0.34\end{array}$ & $\begin{array}{c}100 \\
3.10 \pm 0.08\end{array}$ & $\begin{array}{c}100 \\
3.79 \pm 0.21\end{array}$ & $\begin{array}{c}100 \\
3.96 \pm 0.14\end{array}$ & $\begin{array}{c}100 \\
3.35 \pm 0.07\end{array}$ & $\begin{array}{c}100 \\
3.03 \pm 0.10\end{array}$ & $\begin{array}{c}100 \\
3.22 \pm 0.03\end{array}$ & $\begin{array}{c}100 \\
3.07 \pm 0.08\end{array}$ & $\begin{array}{l}100 \\
3.25\end{array}$ & 0.39 & 2.87 & 3.96 \\
\hline Nonprotein N & $1.45 \pm 0.10$ & $1.47 \pm 0.12$ & $1.50 \pm 0.09$ & $1.77 \pm 0.07$ & $1.92 \pm 0.05$ & $1.68 \pm 0.11$ & $1.15 \pm 0.05$ & $1.30 \pm 0.04$ & $1.38 \pm 0.02$ & 1.51 & 0.24 & 1.15 & 1.92 \\
\hline True Protein & $8.85 \pm 1.22$ & $8.83 \pm 2.05$ & $9.99 \pm 0.19$ & $12.67 \pm 1.58$ & $12.76 \pm 1.12$ & $10.46 \pm 1.06$ & $11.79 \pm 0.28$ & $12.03 \pm 0.30$ & $10.59 \pm 0.40$ & 10.88 & 1.51 & 8.83 & 12.76 \\
\hline Ash & $9.54 \pm 0.63$ & $8.64 \pm 0.26$ & $9.04 \pm 0.12$ & $8.89 \pm 0.20$ & $11.40 \pm 0.11$ & $10.22 \pm 0.08$ & $8.39 \pm 0.41$ & $7.75 \pm 0.26$ & $8.05 \pm 0.21$ & 9.10 & 1.14 & 7.75 & 11.40 \\
\hline Crude fat & $30.31 \pm 0.14$ & $19.03 \pm 0.80$ & $23.33 \pm 0.29$ & $21.40 \pm 0.05$ & $21.44 \pm 0.61$ & $20.57 \pm 0.27$ & $19.07 \pm 2.01$ & - & - & 22.18 & 3.88 & 19.03 & 30.31 \\
\hline Total dietary fiber & $8.41 \pm 0.97$ & $10.43 \pm 1.30$ & $12.09 \pm 1.50$ & $12.56 \pm 1.80$ & $17.69 \pm 6.72$ & $16.40 \pm 6.78$ & $24.43 \pm 2.00$ & $20.44 \pm 1.50$ & - & 15.31 & 5.42 & 8.41 & 24.43 \\
\hline Other solubles ** & $42.84 \pm 1.67$ & $53.07 \pm 3.51$ & $45.42 \pm 1.44$ & $45.20 \pm 0.25$ & $36.86 \pm 7.00$ & $43.05 \pm 7.71$ & $35.94 \pm 2.56$ & - & - & 43.20 & 5.77 & 35.94 & 53.07 \\
\hline
\end{tabular}

* data for each monthly lot represent mean \pm sd of triplicate measurements. Details on the origin of corn feedstock and on lot timings are provided in Materials and Methods and in Table S1; - data not available; ** calculated by difference. 
Table 4. Minerals and trace element contents of thin stillage from the dry-grind corn ethanol plant. Mean, standard deviation, and range of values observed over a one-year period $(n=9)^{*}$.

\begin{tabular}{|c|c|c|c|c|c|c|c|c|c|c|c|c|c|}
\hline & Lot 1 & Lot 2 & Lot 3 & Lot 4 & Lot 5 & Lot 6 & Lot 7 & Lot 8 & Lot 9 & mean & sd & $\min$ & $\max$ \\
\hline & \multicolumn{13}{|c|}{ content per $100 \mathrm{~g}$ wet weight } \\
\hline $\mathrm{K}(\mathrm{mg})$ & $151.69 \pm 2.51$ & $171.55 \pm 2.05$ & $172.78 \pm 0.57$ & $198.70 \pm 3.83$ & $189.29 \pm 5.15$ & $177.84 \pm 7.1$ & $196.48 \pm 8.15$ & $179.68 \pm 1.29$ & $172.20 \pm 5.50$ & 178.91 & 14.52 & 151.69 & 198.70 \\
\hline $\mathrm{P}(\mathrm{mg})$ & $108.78 \pm 1.51$ & $111.38 \pm 4.1$ & $110.27 \pm 1.87$ & $135.10 \pm 2.64$ & $126.25 \pm 2.0$ & $125.23 \pm 1.9$ & $129.17 \pm 5.84$ & $126.99 \pm 8.64$ & $116.71 \pm 4.52$ & 121.10 & 9.50 & 108.78 & 135.10 \\
\hline $\mathrm{Na}(\mathrm{mg})$ & $47.10 \pm 0.52$ & $43.10 \pm 0.82$ & $39.71 \pm 1.53$ & $36.52 \pm 0.43$ & $60.49 \pm 1.1$ & $66.70 \pm 1.66$ & $73.66 \pm 3.58$ & $69.74 \pm 4.73$ & $47.22 \pm 1.59$ & 53.81 & 13.966 & 36.52 & 73.66 \\
\hline $\mathrm{Mg}(\mathrm{mg})$ & $41.02 \pm 0.25$ & $44.55 \pm 0.52$ & $41.07 \pm 3.79$ & $51.42 \pm 1.02$ & $54.04 \pm 0.96$ & $51.63 \pm 1.47$ & $52.30 \pm 2.61$ & $48.34 \pm 3.66$ & $50.43 \pm 1.47$ & 48.31 & 4.92 & 41.02 & 54.04 \\
\hline $\mathrm{Ca}(\mathrm{mg})$ & $4.72 \pm 0.03$ & $5.19 \pm 0.26$ & $4.82 \pm 0.46$ & $5.71 \pm 0.08$ & $6.08 \pm 0.11$ & $5.53 \pm 0.09$ & $6.50 \pm 0.46$ & $7.08 \pm 0.55$ & $6.04 \pm 0.22$ & 5.74 & 0.78 & 4.72 & 7.08 \\
\hline $\mathrm{Fe}(\mathrm{mg})$ & $0.66 \pm 0.02$ & $0.71 \pm 0.02$ & $0.63 \pm 0.01$ & $0.82 \pm 0.02$ & $0.81 \pm 0.02$ & $0.90 \pm 0.03$ & $0.85 \pm 0.06$ & $0.87 \pm 0.07$ & $0.58 \pm 0.02$ & 0.76 & 0.12 & 0.58 & 0.90 \\
\hline Zn (mg) & $0.59 \pm 0.02$ & $0.77 \pm 0.03$ & $0.62 \pm 0.02$ & $0.75 \pm 0.02$ & $0.71 \pm 0.01$ & $0.66 \pm 0.02$ & $0.75 \pm 0.04$ & $0.77 \pm 0.05$ & $0.66 \pm 0.03$ & 0.70 & 0.07 & 0.59 & 0.77 \\
\hline $\mathrm{Mn}(\mathrm{mg})$ & $0.22 \pm 0.01$ & $0.20 \pm 0.01$ & $0.19 \pm 0.01$ & $0.22 \pm 0.01$ & $0.23 \pm 0.01$ & $0.22 \pm 0.01$ & $0.24 \pm 0.01$ & $0.23 \pm 0.02$ & $0.20 \pm 0.01$ & 0.22 & 0.02 & 0.19 & 0.24 \\
\hline \multirow[t]{2}{*}{$\mathrm{Cu}(\mathrm{mg})$} & $0.02 \pm 0.00$ & $0.03 \pm 0.00$ & $0.03 \pm 0.00$ & $0.03 \pm 0.00$ & $0.02 \pm 0.00$ & $0.03 \pm 0.00$ & $0.03 \pm 0.00$ & $0.03 \pm 0.00$ & $0.04 \pm 0.00$ & 0.03 & 0.01 & 0.02 & 0.04 \\
\hline & \multicolumn{13}{|c|}{ content per $100 \mathrm{~g}$ dry weight } \\
\hline $\mathrm{K}(\mathrm{g})$ & $1.97 \pm 0.03$ & $2.15 \pm 0.03$ & $2.20 \pm 0.09$ & $2.35 \pm 0.05$ & $2.78 \pm 0.08$ & $2.23 \pm 0.09$ & $2.25 \pm 0.09$ & $1.81 \pm 0.13$ & $2.01 \pm 0.06$ & 2.19 & 0.27 & 1.81 & 2.78 \\
\hline $\mathrm{P}(\mathrm{g})$ & $1.41 \pm 0.02$ & $1.39 \pm 0.05$ & $1.40 \pm 0.02$ & $1.60 \pm 0.03$ & $1.85 \pm 0.03$ & $1.57 \pm 0.02$ & $1.48 \pm 0.07$ & $1.28 \pm 0.08$ & $1.36 \pm 0.05$ & 1.48 & 0.17 & 1.28 & 1.85 \\
\hline $\mathrm{Na}(\mathrm{g})$ & $0.61 \pm 0.01$ & $0.54 \pm 0.01$ & $0.51 \pm 0.02$ & $0.43 \pm 0.01$ & $0.89 \pm 0.02$ & $0.84 \pm 0.02$ & $0.84 \pm 0.04$ & $0.70 \pm 0.05$ & $0.55 \pm 0.02$ & 0.66 & 0.17 & 0.43 & 0.89 \\
\hline $\mathrm{Mg}(\mathrm{g})$ & $0.53 \pm 0.00$ & $0.56 \pm 0.01$ & $0.52 \pm 0.05$ & $0.61 \pm 0.01$ & $0.79 \pm 0.01$ & $0.65 \pm 0.02$ & $0.60 \pm 0.03$ & $0.49 \pm 0.04$ & $0.59 \pm 0.02$ & 0.59 & 0.09 & 0.49 & 0.79 \\
\hline $\mathrm{Ca}(\mathrm{mg})$ & $61.32 \pm 0.40$ & $64.95 \pm 3.21$ & $61.32 \pm 5.81$ & $67.64 \pm 0.94$ & $89.21 \pm 1.54$ & $69.46 \pm 1.16$ & $74.40 \pm 5.24$ & $71.31 \pm 5.50$ & $70.50 \pm 2.57$ & 70.01 & 8.46 & 61.32 & 89.21 \\
\hline $\mathrm{Fe}(\mathrm{mg})$ & $8.63 \pm 0.21$ & $8.84 \pm 0.22$ & $7.96 \pm 0.15$ & $9.75 \pm 0.24$ & $11.92 \pm 0.28$ & $11.26 \pm 0.37$ & $9.67 \pm 0.68$ & $8.74 \pm 0.71$ & $6.76 \pm 0.24$ & 9.28 & 1.59 & 6.76 & 11.92 \\
\hline $\mathrm{Zn}(\mathrm{mg})$ & $7.72 \pm 0.21$ & $9.61 \pm 0.34$ & $7.95 \pm 0.22$ & $8.83 \pm 0.19$ & $10.41 \pm 0.16$ & $8.27 \pm 0.30$ & $8.62 \pm 0.42$ & $7.77 \pm 0.49$ & $7.69 \pm 0.30$ & 8.54 & 0.94 & 7.69 & 10.41 \\
\hline $\mathrm{Mn}(\mathrm{mg})$ & $2.80 \pm 0.04$ & $2.56 \pm 0.09$ & $2.48 \pm 0.11$ & $2.61 \pm 0.06$ & $3.41 \pm 0.07$ & $2.80 \pm 0.05$ & $2.71 \pm 0.13$ & $2.36 \pm 0.18$ & $2.36 \pm 0.08$ & 2.68 & 0.32 & 2.36 & 3.41 \\
\hline $\mathrm{Cu}(\mathrm{mg})$ & $0.26 \pm 0.01$ & $0.37 \pm 0.02$ & $0.35 \pm 0.01$ & $0.37 \pm 0.01$ & $0.36 \pm 0.02$ & $0.36 \pm 0.01$ & $0.29 \pm 0.02$ & $0.32 \pm 0.02$ & $0.51 \pm 0.02$ & 0.36 & 0.07 & 0.26 & 0.51 \\
\hline
\end{tabular}

* data for each lot represent mean \pm sd of quadruplicate measurements. Details on the origin of corn feedstock and on lot timings are provided in Materials and Methods and in Table S1. 
The fatty acid profile of the lots of thin stillage analyzed is reported in Table 5 . The profile was similar to that observed for corn oil, with linoleic acid (C18:2 n-6) as the prevalent fatty acid (about $50 \%$ of total fatty acids). Palmitic (C16:0) and oleic (C18:1 n-9) acids, accounting altogether for more than $40 \%$ of total fatty acids, were the other two most prominent fatty acids. With respect to corn oil, thin stillage showed a higher percentage of saturated fatty acids, mostly contributed by palmitic acid (21-24\% of total fatty acids). As also observed in corn oil, in thin stillage, the mass spectrum analysis allowed for the identification of minor amounts of ethyl esters of palmitic and linoleic acids, most probably arising from the fermentation process. The values here reported are the sum of ethyl and methyl esters. Fatty acid values are in accordance with other studies [20], although the presence of ethyl esters was not reported before.

The profile of the different lots of thin stillage examined showed a low variability, as indicated by $C V$ values $(<10 \%)$, which indicates a uniform quality of the feedstock as well as standardized conditions of the biotechnological process.

\section{Materials and Methods}

\subsection{Collection of Side-Stream Samples}

Post-fermentation corn oil and thin stillage samples were obtained from the industrial dry-grind bioethanol plant ENVIRAL a.s. (Leopoldov, Slovakia). A yellow non-genetically modified corn (Zea mays), grown in the Central East Europe region, was the feedstock processed. The study was conducted in the period June 2018-June 2019 (Table S1). The first side stream samples (Lot 1) were obtained from corn harvested in the preceding year 2017. The new harvest season started in September 2018 and by October 2018 (Lot 2) the newly harvested corn was utilized for the bioethanol production process.

Post-fermentation corn oil, obtained by centrifugation of corn syrup, was taken from a plate spinner located under the oil flow control and stored in a sedimentation tank to eliminate any particles that tend to settle and precipitate while cooling down. Thereafter, corn oil samples were put into clean plastic containers and kept in a dry place, away from heat and light sources.

Thin stillage, currently concentrated by evaporation, was sampled from the discharge valve located in the evaporator compartments of the commercial dry-grind ethanol facility.

\subsection{Treatment of Samples}

Corn oil samples collected for daily routine analyses (water content, sedimentation, total contamination, acid value, minerals) and thin stillage samples for basic check of $\mathrm{pH}$ and dry matter, were immediately taken to the ENVIRAL's in house laboratory without any further treatment.

On a monthly basis (approximately every 30-45 days), $1 \mathrm{~L}$ of corn oil (liquid) and $3 \mathrm{~L}$ of thin stillage (frozen) were delivered (24-48 h from collection) to CREA laboratory (Food and Nutrition Research Centre, Rome, Italy) for in-depth characterization of macronutrients, mineral elements, and fatty acids. Thin stillage samples had been frozen by ENVIRAL straight after sampling, prior to transport, with the intention of preventing any undesirable secondary fermentation during shipment; slow melting during transportation without any changes of thin stillage properties was acceptable, allowing immediate processing at CREA's lab. 
Table 5. Fatty acid profile of thin stillage from the dry-grind corn ethanol plant. Mean, standard deviation, and range of values observed over a one-year period ( $\mathrm{n}=7$ ) *. Values are expressed as percent of total fatty acids.

\begin{tabular}{|c|c|c|c|c|c|c|c|c|c|c|c|}
\hline & Lot 1 & Lot 2 & Lot 3 & Lot 4 & Lot 5 & Lot 6 & Lot 7 & mean & sd & $\min$ & $\max$ \\
\hline & \multicolumn{11}{|c|}{$\%$ of total fatty acids } \\
\hline Lauric acid (C12:0) & $0.00 \pm 0.00$ & $0.02 \pm 0.00$ & $0.02 \pm 0.01$ & $0.00 \pm 0.00$ & $0.03 \pm 0.00$ & $0.06 \pm 0.03$ & $0.00 \pm 0.00$ & 0.02 & 0.02 & 0.00 & 0.06 \\
\hline Myristic acid (C14:0) & $0.08 \pm 0.02$ & $0.06 \pm 0.02$ & $0.09 \pm 0.00$ & $0.06 \pm 0.02$ & $0.12 \pm 0.02$ & $0.15 \pm 0.04$ & $0.12 \pm 0.02$ & 0.10 & 0.03 & 0.06 & 0.15 \\
\hline Pentadecylic acid (C15:0) & $0.00 \pm 0.00$ & $0.03 \pm 0.01$ & $0.02 \pm .000$ & $0.00 \pm 0.00$ & $0.00 \pm 0.00$ & $0.00 \pm 0.00$ & $0.00 \pm 0.00$ & 0.01 & 0.01 & 0.00 & 0.03 \\
\hline Palmitic acid (C16:0) & $23.29 \pm 0.30$ & $23.00 \pm 0.37$ & $22.12 \pm 0.70$ & $24.10 \pm 0.47$ & $23.28 \pm 0.38$ & $22.17 \pm 2.39$ & $21.40 \pm 0.66$ & 22.76 & 0.91 & 21.40 & 24.10 \\
\hline Palmitoleic acid (C16:1 n-7) & $0.00 \pm 0.00$ & $0.09 \pm 0.03$ & $0.06 \pm 0.01$ & $0.05 \pm 0.00$ & $0.07 \pm 0.01$ & $0.00 \pm 0.00$ & $0.00 \pm 0.00$ & 0.04 & 0.04 & 0.00 & 0.09 \\
\hline Margaric acid (C17:0) & $0.00 \pm 0.00$ & $0.07 \pm 0.00$ & $0.10 \pm 0.01$ & $0.00 \pm 0.00$ & $0.00 \pm 0.00$ & $0.00 \pm 0.00$ & $0.00 \pm 0.00$ & 0.03 & 0.05 & 0.00 & 0.10 \\
\hline Stearic acid (C18:0) & $3.31 \pm 0.07$ & $3.16 \pm 0.08$ & $3.32 \pm 0.03$ & $3.48 \pm 0.41$ & $2.49 \pm 0.06$ & $1.37 \pm 0.19$ & $1.77 \pm 0.14$ & 2.70 & 0.85 & 1.37 & 3.48 \\
\hline Oleic acid (C18:1 n-9) & $21.08 \pm 0.50$ & $21.35 \pm 0.54$ & $21.20 \pm 0.67$ & $20.36 \pm 1.10$ & $21.13 \pm 0.17$ & $24.79 \pm 1.42$ & $23.73 \pm 0.47$ & 21.95 & 1.64 & 20.36 & 24.79 \\
\hline Linoleic acid (C18:2 n-6) & $49.14 \pm 0.30$ & $49.79 \pm 0.32$ & $50.76 \pm 0.17$ & $49.48 \pm 1.07$ & $50.55 \pm 0.29$ & $49.35 \pm 1.32$ & $50.95 \pm 0.05$ & 50.00 & 0.74 & 49.14 & 50.95 \\
\hline$\alpha$-Linolenic acid (C18:3n-3) & $1.75 \pm 0.05$ & $1.82 \pm 0.05$ & $1.63 \pm 0.08$ & $1.92 \pm 0.15$ & $1.65 \pm 0.04$ & $1.40 \pm 0.11$ & $1.75 \pm 0.05$ & 1.70 & 0.17 & 1.40 & 1.92 \\
\hline Arachidic acid (C20:0) & $0.16 \pm 0.02$ & $0.32 \pm 0.02$ & $0.34 \pm 0.02$ & $0.25 \pm 0.07$ & $0.30 \pm 0.03$ & $0.26 \pm 0.04$ & $0.29 \pm 0.05$ & 0.27 & 0.06 & 0.16 & 0.34 \\
\hline Gondoic acid (C20:1 n-9) & $0.39 \pm 0.04$ & $0.18 \pm 0.11$ & $0.28 \pm 0.07$ & $0.00 \pm 0.00$ & $0.26 \pm 0.03$ & $0.41 \pm 0.04$ & $0.00 \pm 0.00$ & 0.25 & 0.15 & 0.00 & 0.41 \\
\hline Eicosadienoic acid (C20:2n-6) & $0.00 \pm 0.00$ & $0.07 \pm 0.01$ & $0.09 \pm 0.03$ & $0.29 \pm 0.08$ & $0.00 \pm 0.00$ & $0.00 \pm 0.00$ & $0.00 \pm 0.00$ & 0.08 & 0.11 & 0.00 & 0.29 \\
\hline Behenic acid (C22:0) & $0.00 \pm 0.00$ & $0.13 \pm 0.01$ & $0.13 \pm 0.00$ & $0.00 \pm 0.00$ & $0.14 \pm 0.02$ & $0.06 \pm 0.04$ & $0.00 \pm 0.00$ & 0.09 & 0.06 & 0.00 & 0.14 \\
\hline Total SFA & $26.84 \pm 0.50$ & $26.69 \pm 0.52$ & $26.03 \pm 0.70$ & $27.90 \pm 0.90$ & $26.35 \pm 0.45$ & $24.06 \pm 2.66$ & $23.57 \pm 0.46$ & 25.92 & 1.56 & 23.57 & 27.90 \\
\hline Total MUFA & $21.47 \pm 0.40$ & $21.63 \pm 0.43$ & $21.53 \pm 0.60$ & $20.41 \pm 1.10$ & $21.46 \pm 0.17$ & $25.19 \pm 1.38$ & $23.73 \pm 0.47$ & 22.20 & 1.65 & 20.41 & 25.19 \\
\hline Total PUFA & $50.89 \pm 0.30$ & $51.69 \pm 0.28$ & $52.48 \pm 0.26$ & $51.69 \pm 1.11$ & $52.20 \pm 0.32$ & $50.75 \pm 1.43$ & $52.70 \pm 0.02$ & 51.77 & 0.75 & 50.75 & 52.70 \\
\hline Total n-6 PUFA & $49.14 \pm 0.30$ & $49.87 \pm 0.32$ & $50.85 \pm 0.18$ & $49.77 \pm 1.11$ & $50.55 \pm 0.29$ & $49.35 \pm 1.32$ & $50.95 \pm 0.05$ & 50.07 & 0.72 & 49.14 & 50.95 \\
\hline Total n-3 PUFA & $1.75 \pm 0.05$ & $1.82 \pm 0.05$ & $1.63 \pm 0.08$ & $1.92 \pm 0.15$ & $1.65 \pm 0.04$ & $1.40 \pm 0.11$ & $1.75 \pm 0.05$ & 1.70 & 0.17 & 1.40 & 1.92 \\
\hline n-6/n-3 PUFA ratio & $28.08 \pm 0.70$ & $27.43 \pm 0.88$ & $31.29 \pm 1.42$ & $26.01 \pm 2.26$ & $30.61 \pm 0.69$ & $35.35 \pm 1.84$ & $29.07 \pm 0.82$ & 29.69 & 3.08 & 26.01 & 35.35 \\
\hline PUFA/SFA ratio & $1.90 \pm 0.05$ & $1.94 \pm 0.05$ & $2.02 \pm 0.06$ & $1.85 \pm 0.09$ & $1.98 \pm 0.05$ & $2.13 \pm 0.31$ & $2.24 \pm 0.04$ & 2.01 & 0.14 & 1.85 & 2.24 \\
\hline
\end{tabular}

data for each lot represent mean $\pm \mathrm{sd}$ of triplicate measurements. Details on the origin of corn feedstock and on lot timings are provided in Materials and Methods and in Table S1. 
Samples for in-depth chemical characterization, upon arrival at the CREA premises, were immediately refrigerated $\left(+4{ }^{\circ} \mathrm{C}\right)$ and treated as accurately as possible in order to preserve their original properties until analyses. Corn oil was preserved from light and heat and analyzed without any pre-treatment. Before sampling for analyses, the oil was brought to room temperature and gently shaken in order to re-suspend any solid material sedimented at the bottom of the bottle. Thin stillage was analyzed as received, in the liquid state and, when analytical methods required concentrated samples (i.e., fat, minerals, dietary fiber), after lyophilization. The consistency of results obtained on liquid and freeze-dried samples was checked and confirmed by replicate and parallel analyses on freeze-dried and liquid aliquots of thin stillage. Proximate composition, nonprotein nitrogen, total dietary fiber, mineral content, and fatty acid profile were the parameters studied.

\subsection{Analytical Methods}

\subsubsection{Daily Routine Analyses}

Water content in corn oil was determined by the Karl-Fisher titration method according to EN ISO 12937 [30]. Sedimentation in corn oil was measured from homogenized sample after centrifugation. The amount of separated material, the sediment, was volumetrically measured in a calibrated centrifuge tube. Total contamination was controlled by gravimetric method according to EN 12662 [31]. A pre-weighed membrane filter was used for the vacuum filtration of a known volume of tested sample, washed, dried, and weighed. Acid value was determined by non-aqueous potentiometric acid-base titration with an alcoholic solution of potassium hydroxide according to EN 14104 [32]. Mineral contents (phosphorus, calcium, magnesium, sodium, and potassium) were determined by inductively coupled plasma (ICP) emission spectrometry according to EN 14107 [33].

Thin stillage was routinely checked for $\mathrm{pH}$ by potentiometric measurement using a glass electrode and dry matter was determined by weighing after the drying of pre-dried sample in an oven at $130{ }^{\circ} \mathrm{C}$.

\subsubsection{Proximate Composition}

Moisture, crude protein, crude fat, and ash contents were determined according to the Association of Official Analytical Chemists's (AOAC) [34] methods. Total nitrogen was evaluated by the Kjeldahl procedure. Nonprotein nitrogen (NPN) was determined following the Kjeldahl method after protein precipitation with $10 \%(\mathrm{w} / \mathrm{v})$ trichloroacetic acid and filtration. True protein $\mathrm{N}$ was calculated as the difference between total nitrogen and NPN. The true protein content was calculated by multiplying true protein $\mathrm{N}$ by the factor 6.25 . The crude fat content was determined by Soxhlet extraction. Ash content was determined by incineration in a muffle furnace at $550{ }^{\circ} \mathrm{C}$. Total dietary fiber was determined according to the official enzymatic-gravimetric method [35]. Available carbohydrates, organic acids, and other solubles were calculated by differences. All analyses were performed in triplicate. Results were normalized both on a wet mass and dry mass basis.

\subsubsection{Minerals and Trace Elements}

Minerals $(\mathrm{Ca}, \mathrm{Mg}, \mathrm{Na}, \mathrm{K}, \mathrm{P})$ and trace elements $(\mathrm{Fe}, \mathrm{Zn}, \mathrm{Cu}, \mathrm{Mn})$ contents of thin stillage were quantified by inductively coupled plasma optical emission spectrometry (Optima 8000 ${ }^{\mathrm{TM}}$ ICP-OES, Perkin-Elmer, Waltham, MA, USA) after liquid ashing $\left(6 \mathrm{~mL} \mathrm{HNO}_{3}+1 \mathrm{~mL} \mathrm{H}_{2} \mathrm{O}_{2}\right)$ of lyophilized thin stillage in a microwave digestion system (1200 Mega, Milestone srl, Italy). Standard reference materials, peanut butter (SRM 2387, National Institute of Standards and Technology), cabbage (IAEA-359, International Atomic Energy Agency Reference Materials Group), and haricots vert (BCR 383, Community Bureau of Reference, Brussels), were analyzed as a check on the accuracy of the analysis. Analyses were performed in quadruplicate. Data were normalized both on a wet mass and dry mass basis considering the water content of liquid thin stillage (90-93\%). 


\subsubsection{Total Lipids and Fatty Acids}

Total lipids were extracted from lyophilized thin stillage samples according to the method of Bligh and Dyer [36]. In both samples of untreated crude corn oil and thin stillage lipid extracts the fatty acids were methylated using boron trifluoride in methanol as esterification reagent [37]. Separations were accomplished on a Mega-wax column $(30 \mathrm{~m} \times 0.32 \mathrm{~mm}$ inner diameter, $0.25 \mu \mathrm{m}$ film thickness). The esterified fatty acids were identified and quantified by GC-MS-FID (7890A Series-Agilent Technologies Santa Clara, CA, USA). Fatty acids were identified comparing retention times with known authentic standards and using the NIST08 Mass Spectral Library (National Institute of Standards and Technology, Gaithersburg, MD, USA). FAME Mix C4-C24 (Supelco, Bellofonte, PA, USA) was executed as a control of the accuracy of the analysis. Analyses were performed in triplicate. Data are reported as percent of total fatty acids.

\subsection{Data Treatment}

For each parameter, analyses of individual lots of post-fermentation corn oil and thin stillage were performed at least in triplicate. Data for single lots, mean, standard deviation, coefficient of variation, and range of values detected during the experimental period were calculated with Microsoft Excel software, 2013 version.

\section{Conclusions}

In conclusion, in this study the complete monthly data, measured during a one-year period, here presented, describe macronutrient, fatty acid and mineral profiles of post-fermentation corn oil and thin stillage from commercial bioethanol production. These data provide basic indications on the main properties and qualities of the two side streams. In particular, the high linoleic and oleic acids concentrations, fatty acids of nutritional interest, and the relatively high and constant presence of minerals and macronutrients in the stillage suggest a biotechnological utilization. The low variability of the chemical characteristics observed during the year in the two side streams, in spite of the different origin and seasonality of corn feedstock lots and of the complex biotechnological processes, represent an important element for their industrial utilization.

For a full exploitation of the potentialities of the side streams in this study, a detailed investigation on the bioactive compounds present therein and a sustainability assessment of recovery processes represent the next necessary steps of this study.

The concomitant presence in corn bioethanol co-products of a variegate complex of molecules of nutritional interest, with several different potential high-value market applications, make the perspective of their recovery a promising strategy to maximize the sustainability of corn dry-grind bioethanol biorefineries and create new cross-sector interconnections according to circular economy principles. The aim of the Project is to fractionate the post-fermentation corn oil prior to non-edible biodiesel applications, to obtain desirable valuable molecules, and simultaneously optimize feedstock streams for biodiesel application by removing these molecules.

Supplementary Materials: The following are available online, Figure S1: Scheme of grain to ethanol process and its connection to the EXCornsEED project. Table S1: Sampling date at ENVIRAL's plants of the different side stream lots analysed in the study.

Author Contributions: Conceptualization, G.D.L. and P.O.; writing-original draft preparation, G.D.L.; writing-review and editing, G.D.L. and P.O.; investigation, A.A., R.C., I.C., V.C., S.F.N., P.G. and M.L.; formal analysis, J.S.d.P. and T.J.; Supervision, G.D.L.; Data curation and visualization, G.D.L., P.O. and V.C.; Validation, G.D.L. and G.L.B.; Funding acquisition, G.D.L. and P.O. All authors have read and agreed to the published version of the manuscript.

Funding: This study was carried out in the frame of the EXCornsEED project. This project received funding from the Bio Based Industries Joint Undertaking under the European Union's Horizon 2020 research and innovation program under grant agreement $n^{\circ} 792054$.

Conflicts of Interest: The authors declare no conflict of interest. 


\section{References}

1. European Commission. Consolidated version of the Treaty on the Functioning of the European Union. Official Journal of the European Union, 26 October 2012; C326/47.

2. European Economic and Social Committee. Communication from the Commission to the European Parliament, the Council, the European Economic and Social Committee, the Committee of the Regions and the European Investment Bank a Framework Strategy for a Resilient Energy Union with a Forward-Looking Climate Change Policy. Available online: https://eur-lex.europa.eu/legal-content/EN/TXT/?uri=CELEX: 52015DC0080 (accessed on 23 July 2020).

3. European Economic and Social Committee. Communication from the Commission to the European Parliament and the Council Achieving the 10\% Electricity Interconnection Target Making Europe's Electricity Grid Fit for 2020. Available online: https://eur-lex.europa.eu/legal-content/EN/TXT/?uri=COM\%3A2015\%3A82\%3AFIN (accessed on 23 July 2020).

4. European Commission. Directive 2009/28/EC of the European Parliament and of the Council of 23 April 2009 on the promotion of the use of energy from renewable sources and amending and subsequently repealing Directives 2001/77/EC and 2003/30/EC. Official Journal of the European Union, 6 June 2009; L140/16.

5. European Commission. Directive 2009/30/EC of The European Parliament and of the Council of 23 April 2009 amending Directive 98/70/EC as regards the specification of petrol, diesel and gas-oil and introducing a mechanism to monitor and reduce greenhouse gas emissions and amending Council Directive 1999/32/EC as regards the specification of fuel used by inland waterway vessels and repealing Directive 93/12/EEC, 2009. Official Journal of the European Union, 5 June 2009; L144/88.

6. European Commission. Directive (EU) 2015/1513 of the European Parliament and of the Council of 9 September 2015 amending Directive 98/70/EC relating to the quality of petrol and diesel fuels and amending Directive 2009/28/EC on the promotion of the use of energy from renewable sources. Official Journal of the European Union, 15 September 2015; L239/1.

7. European Commission. Renewable Energy Progress Report. Report from the Commission to the European Parliament, the Council, the European Economic and Social Committee and the Committee of the Regions; European Commission: Brussels, Belgium, 2017.

8. ePURE, European Renewable Ethanol. Statistics and Infographics 2018. Share of European Renewable Ethanol Produced from Each Feedstock Type. Available online: https:/epure.org/media/1930/share-of-europeanrenewable-ethanol-produced-from-each-feedstock-type-2018.jpg (accessed on 30 July 2020).

9. ePURE, European Renewable Ethanol. Statistics and Infographics 2018. European Renewable Ethanol Installed Production Capacity. Available online: https://epure.org/media/1925/european-renewable-ethanol-installedproduction-capacity-2018.jpg (accessed on 30 July 2020).

10. European Commission. A Sustainable Bioeconomy for Europe. Strengthening the Connection between Economy, Society and the Environment. Updated Bioeconomy Strategy 2018; Directorate-General for Research and Innovation: Brussels, Belgium, 2018.

11. European Commission. Communication from the Commission to the European Parliament, the Council, the European Economic and Social Committee and the Committee of the Regions. A New Circular Economy Action Plan for a Cleaner and More Competitive Europe; European Commission: Brussels, Belgium, 2020.

12. Kim, Y.; Mosier, N.; Hendrickson, R.; Ezeji, T.; Blaschek, H.; Dien, B.; Dale, B.; Ladisch, M. Composition of corn dry-grind ethanol by-products: DDGS, wet cake, and thin stillage. Bioresour. Technol. 2008, 99, 5165-5176. [CrossRef] [PubMed]

13. Reis, C.E.R.; Rajendran, A.; Hu, B. New technologies in value addition to the thin stillage from corn-to-ethanol process. Rev. Environ. Sci. Biotechnol. 2017, 16, 175-206. [CrossRef]

14. Ferreira, J.A.; Lennartsson, P.R.; Taherzadeh, M.J. Production of ethanol and biomass from thin stillage by Neurospora intermedia: A pilot study for process diversification. Eng. Life Sci. 2015, 15, 751-759. [CrossRef]

15. Pietrzak, W.; Kawa-Rygielska, J. Backset valorization in dry-grind ethanol process by co-culture of edible filamentous fungi and fodder yeast. J. Clean. Prod. 2019, 220, 376-385. [CrossRef]

16. Mitra, D.; van Leeuwen, J.; Lamsal, B. Heterotrophic/mixotrophic cultivation of oleaginous Chlorella vulgaris on industrial co-products. Algal Res. 2012, 1, 40-48. [CrossRef]

17. Beigbeder, J.B.; Boboescu, J.Z.; Lavoie, J.M. Thin stillage treatment and co-production of bio-commodities through finely tuned Chlorella vulgaris cultivation. J. Clean. Prod. 2019, 216, 257-267. [CrossRef] 
18. Dowd, M.K.; Reilly, P.J.; Trahanovsky, W.S. Low molecular weight organic composition of ethanol stillage from corn. Cereal Chem. 1993, 70, 204-209.

19. Moreau, R.A.; Hicks, K.B.; Johnston, D.B.; Laun, N.P. The Composition of Crude Corn Oil Recovered after Fermentation via Centrifugation from a Commercial Dry Grind Ethanol Process. J. Am. Oil Chem. Soc. 2010, 87, 895-902. [CrossRef]

20. Moreau, R.A.; Liu, K.; Winkler-Moser, J.K.; Singh, V. Changes in lipid composition during dry grind ethanol processing of corn. J. Am. Oil Chem. Soc. 2011, 88, 435-442. [CrossRef]

21. Ratanapariyanuch, K.; Shen, J.; Jia, Y.; Tyler, R.T.; Shim, Y.Y.; Reaney, M.J.T. Rapid NMR Method for the Quantification of Organic Compounds in Thin Stillage. J. Agric. Food Chem. 2011, 59, 10454-10460. [CrossRef] [PubMed]

22. Winkler-Moser, J.K.; Breyer, L. Composition and oxidative stability of crude oil extracts of corn germ and distillers grains. Ind. Crops Prod. 2011, 33, 572-578. [CrossRef]

23. Wood, C.; Rosentrater, K.A.; Muthukumarappan, K.; Gu, Z. Quantification of physical and chemical properties and identification of potentially valuable components from fuel ethanol process streams. Cereal Chem. 2013, 90, 70-79. [CrossRef]

24. Banga, S.; Varshney, P. Effect of impurities on performance of biodiesel: A review. J. Sci. Ind. Res. 2010, 69, 575-579.

25. Kai, T.; Kubo, A.; Nakazato, T.; Takanashi, H.; Uemura, Y. Biomass and renewables influence of the acid value on biodiesel fuel production using a two-step batch process with a homogeneous catalyst. Int. J. Biomass Renew. 2012, 1, 15-20.

26. Gerpen, J.H.; He, B.B. Biodiesel and Renewable Diesel Production Methods. In Advances in Biorefineries: Biomass and Waste Supply Chain Exploitation; Waldron, K., Ed.; Woodhead Publishing, Elsevier: Cambridge, UK, 2014.

27. Alves, B.; Carvalho, F.; Cruz, A.; Filho, H.; Dantas, K. Determination of Ca, Mg, Na, and K in Biodiesel of Oilseed from Northern Brazil. Rev. Virtual Quim. 2018, 10, 542-550. [CrossRef]

28. CREA. Italian Food Composition Tables; Research Centre for Food and Nutrition: Rome, Italy; Available online: https://www.alimentinutrizione.it/tabelle-nutrizionali/009660 (accessed on 25 July 2020).

29. Barrera-Arellano, D.; Badan-Ribeiro, A.P.; Serna-Saldivar, S.O. Chapter 21-Corn Oil: Composition, Processing, and Utilization. In Corn: Chemistry and Technology, 3rd ed.; Serna-Saldivar, S.O., Ed.; AACC International Press: Duxford, UK, 2019; pp. 593-613, ISBN 9780128118863.

30. EN ISO 12937/2000 Petroleum Products-Determination of Water-Coulometric Karl Fischer Titration Method.

31. EN 12662/2014 Liquid Petroleum Products-Determination of total contamination in middle distillates, diesel fuels and fatty acid methyl esters.

32. EN 14104/2003 Fat and oil derivatives. Fatty acid methyl esters (FAME). Determination of acid value.

33. EN 14107/2003 Fat and oil derivatives. Fatty acid methyl esters (FAME). Determination of phosphorous content by inductively coupled plasma (ICP) emission spectrometry.

34. Association of Official Analytical Chemists. Official Methods of Analysis, 18th ed.; Association of Official Analytical Chemists: Arlington, VA, USA, 2005.

35. Association of Official Analytical Chemists. Official Methods of Analysis, 16th ed.; Association of Official Analytical Chemists: Arlington, VA, USA, 1999.

36. Bligh, E.G.; and Dyer, W.J. A rapid method of total lipid extraction and purification. Can. J. Biochem. Physiol. 1959, 37, 911-917. [CrossRef] [PubMed]

37. Metcalfe, L.D.; Schmitz, A.A. The rapid preparation of fatty acid esters for gas chromatographic analysis. Anal. Chem. 1961, 33, 363-364. [CrossRef]

Sample Availability: Samples of the compounds are not available from the authors. 